\title{
BMJ Open Community-based HPV self-collection versus visual inspection with acetic acid in Uganda: a cost-effectiveness analysis of the ASPIRE trial
}

\author{
Alex K Mezei, ${ }^{1}$ Heather N Pedersen, ${ }^{2}$ Stephen Sy, ${ }^{3}$ Catherine Regan, ${ }^{3}$ \\ Sheona M Mitchell-Foster, ${ }^{4}$ Josaphat Byamugisha, ${ }^{5,6}$ Musa Sekikubo, ${ }^{5,6}$ \\ Heather Armstrong, ${ }^{1}$ Angeli Rawat, ${ }^{2}$ Joel Singer, ${ }^{7,8}$ Gina S Ogilvie, ${ }^{2}$ Jane J Kim, ${ }^{3}$ \\ Nicole G Campos ${ }^{3}$
}

To cite: Mezei AK, Pedersen HN, Sy $\mathrm{S}$, et al. Communitybased HPV self-collection versus visual inspection with acetic acid in Uganda: a cost-effectiveness analysis of the ASPIRE trial. BMJ Open 2018;8:e020484. doi:10.1136/ bmjopen-2017-020484

- Prepublication history and additional material for this paper are available online. To view these files, please visit the journal online (http://dx.doi. org/10.1136/bmjopen-2017020484).

Received 9 November 2017 Revised 12 April 2018 Accepted 18 April 2018

Check for updates

For numbered affiliations see end of article.

Correspondence to

Alex K Mezei;

alex.k.mezei@gmail.com

\section{ABSTRACT}

Background Cervical cancer is the leading cause of cancer death for women in Uganda, despite the potential for prevention through organised screening. Communitybased self-collected human papillomavirus (HPV) testing has been proposed to reduce barriers to screening. Objective Our objective was to evaluate the costeffectiveness of the Advances in Screening and Prevention of Reproductive Cancers (ASPIRE) trial, conducted in Kisenyi, Uganda in April $2014(n=500)$. The trial compared screening uptake and compliance with follow-up in two arms: (1) community-based (ie, home or workplace) selfcollected HPV testing (facilitated by community health workers) with clinic-based visual inspection with acetic acid (VIA) triage of HPV-positive women ('HPV-VIA') and (2) clinic-based VIA ('VIA'). In both arms, VIA was performed at the local health unit by midwives with VIA-positive women receiving immediate treatment with cryotherapy.

Design We informed a Monte Carlo simulation model of HPV infection and cervical cancer with screening uptake, compliance and retrospective cost data from the ASPIRE trial; additional cost, test performance and treatment effectiveness data were drawn from observational studies. The model was used to assess the cost-effectiveness of each arm of ASPIRE, as well as an HPV screen-and-treat strategy ('HPV-ST') involving community-based selfcollected HPV testing followed by treatment for all HPVpositive women at the clinic.

Outcome measures The primary outcomes were reductions in cervical cancer risk and incremental costeffectiveness ratios (ICERs), expressed in dollars per year of life saved (YLS).

Results HPV-ST was the most effective and cost-effective screening strategy, reducing the lifetime absolute risk of cervical cancer from $4.2 \%$ (range: $3.8 \%-4.7 \%$ ) to $3.5 \%$ (range: $3.2 \%-4 \%$ ), $2.8 \%$ (range: $2.4 \%-3.1 \%$ ) and $2.4 \%$ (range: $2.1 \%-2.7 \%$ ) with ICERs of US $\$ 130$ (US\$110US\$150) per YLS, US\$240 (US\$210-US\$280) per YLS, and US\$470 (US\$410-US\$550) per YLS when performed one, three and five times per lifetime, respectively. Findings were robust across sensitivity analyses, unless HPV costs were more than quadrupled.

Conclusions Community-based self-collected HPV testing followed by treatment for HPV-positive women has the

\section{Strengths and limitations of this study}

- To the best of our knowledge, this is the first study to leverage randomised trial data to evaluate the cost-effectiveness of community-based self-collected HPV testing that is performed at women's homes or places of work.

- The use of community health workers has the potential to reduce demands on overburdened healthcare providers.

- Costs of the Advances in Screening and Prevention of Reproductive Cancers trial are retrospective as the trial was not designed to evaluate cost-effectiveness. Therefore, costs may reflect study as opposed to real-world conditions.

- We also did not consider human resource and capacity constraints in the Ugandan healthcare system.

potential to be an effective and cost-effective screening strategy.

\section{INTRODUCTION}

High-income countries have significantly reduced cervical cancer incidence and mortality by implementing organised cytology-based screening programmes. ${ }^{1}$ The introduction of the prophylactic HPV vaccine has the potential to further reduce incidence and mortality of cervical and other HPV-related cancers. Despite these advances, cervical cancer remains a major cause of morbidity and mortality in low- and middle-income countries (LMICs) due to the absence of effective, organised screening programmes. In Uganda, cervical cancer is the top cause of cancer death in women. ${ }^{2}$ In 2012, Uganda introduced a publicly funded national HPV vaccination programme in parts of the country. ${ }^{3}$ However, due to low vaccine coverage and the targeting of adolescent girls 
before sexual debut, ${ }^{3}$ screening continues to be the only form of prevention for most women.

There are significant barriers to access, implementation and quality assurance for cytology-based screening in LMICs. A systematic review of cost-effectiveness analyses in LMICs found that cytology is not an efficient screening test in low-resource settings. ${ }^{4}$ The WHO no longer recommends cytology-based screening in LMICs that have not already achieved high coverage and quality assurance. ${ }^{5}$ Instead, visual inspection with acetic acid (VIA) and HPV DNA testing have been proposed as alternatives that may be better suited to low-income settings. While VIA is inexpensive, provides same-visit results and allows for immediate treatment, test performance varies widely across providers, populations and settings. ${ }^{6}$ Currently, WHO recommends HPV testing if resources permit. ${ }^{5} \mathrm{HPV}$ testing can be administered using provider-collected or self-collected sampling. While provider collection (of cervical samples) has been demonstrated to have higher sensitivity to detect precancer, ${ }^{6}$ self-collection (of vaginal samples) has been shown to reduce patient barriers to screening ${ }^{7}$ and may increase effectiveness and cost-effectiveness when it increases population coverage. ${ }^{48}$

While several studies have evaluated the cost-effectiveness of self-collected HPV testing in LMICs, ${ }^{4910}$ we could not identify any that evaluate the cost-effectiveness of a delivery model where self-collected HPV tests are offered at women's homes and/or places of work. In addition, we could not identify any studies that leveraged randomised trial data to test the hypothesis that the increased uptake associated with self-collection is cost-effective relative to clinic-based screening. Therefore, our objective with this study was to evaluate the cost-effectiveness of a randomised trial run by the Advances in Screening and Prevention of Reproductive Cancers (ASPIRE) Project, which compared community-based self-collected HPV testing to clinic-based VIA in Uganda. ${ }^{11}$

\section{METHODS}

\section{Analytical framework}

We used a previously developed Monte Carlo simulation model of HPV infection and cervical cancer that was calibrated to epidemiological data from Uganda ${ }^{10} 1213$ to project the lifetime health and economic outcomes associated with self-collected HPV testing versus clinic-based VIA. The model was informed by screening uptake, follow-up and retrospective costing data from the ASPIRE trial, conducted in Kisenyi, Uganda. ${ }^{11}$

The primary outcomes were the incremental cost-effectiveness ratio (ICER) and per cent reduction in lifetime cervical cancer risk (ie, cumulative lifetime cervical cancer incidence). ICERs are defined as the marginal cost (discounted per women cost) divided by the marginal benefit (discounted life expectancy) of a screening strategy compared with the next most costly strategy, after eliminating strategies that are dominated (defined as either more costly and less effective or having a higher
ICER than more effective strategies). ICERs are expressed in 2014 US\$ per year of life saved (\$/YLS). We considered screening strategies with an ICER below US $\$ 730,{ }^{14}$ the GDP per capita of Uganda in 2014, to be very cost-effective. ${ }^{15}$ In keeping with guidelines on cost-effectiveness analysis, we discounted all costs and future life years at a rate of $3 \%$ per year and evaluated costs from a societal perspective, including costs irrespective of the payer. ${ }^{16}$

\section{The ASPIRE trial}

The ASPIRE trial was conducted in the Kisenyi district of Kampala, Uganda. For the trial, community health workers (CHWs) recruited 500 women at their homes or places of work who were between the ages of 30 and 65 , lived and/or worked in Kisenyi, and had access to a mobile phone. ${ }^{11}$ At the time of enrolment, consenting women were randomised into either HPV self-collection with VIA triage for HPV-positive women ('HPV-VIA') or VIA screen and treat ('VIA'). Women randomised to the HPV-VIA arm received instructions from a CHW on self-collection and were given the opportunity to self-collect HPV, Neisseria gonorrhoea and Chlamydia trachomatis (NGCT) samples. CHWs would then transport the samples to local laboratories in Kampala, Uganda where they were tested for HPV and NGCT. The CHWs contacted women by phone with their results. If HPV-positive, women were scheduled for a VIA triage test by a midwife at Kisenyi Health Unit. If VIA-positive, the women were offered immediate cryotherapy (if eligible for cryotherapy) or sent to a tertiary care centre for further diagnosis and treatment (if ineligible for cryotherapy for reasons such as lesion size, inability to adequately visualise the cervix or suspicion of cancer).

Women randomised to the VIA arm were scheduled for an appointment at the Kisenyi health unit. The CHWs contacted women in this arm by phone to remind them of their VIA appointment. When the VIA was performed, women were also offered provider-collected NGCT testing. Like the HPV-VIA arm, women who had a positive VIA screen were offered immediate cryotherapy (if eligible) or referred to a tertiary care centre for further evaluation and treatment.

\section{Mathematical simulation model}

The individual-based microsimulation model of cervical cancer has been previously described, but we summarise key features here. ${ }^{1012}$ The model was programmed in $\mathrm{C}++$. Girls enter the model at age 9 , and each month face probabilities of transitioning between mutually exclusive health states including HPV infection (stratified by HPV genotype), cervical intraepithelial neoplasia grade 2 (CIN2), CIN3, cervical cancer (local, regional or distant stage) and death (figure 1). Transitions between health states may be determined by age, HPV type, duration of HPV infection, duration of CIN, history of previous infection and patterns of screening and treatment of precancer. Each month, death can occur from cervical cancer or non-cervical cancer causes (ie, background 


\section{Screening, diagnosis and treatment of cervical pre-cancer and cancer}

Screening parameters include coverage, interval, sensitivity and specificity of screening and diagnostic tools, treatment successes and failures, and loss-to-follow-up.

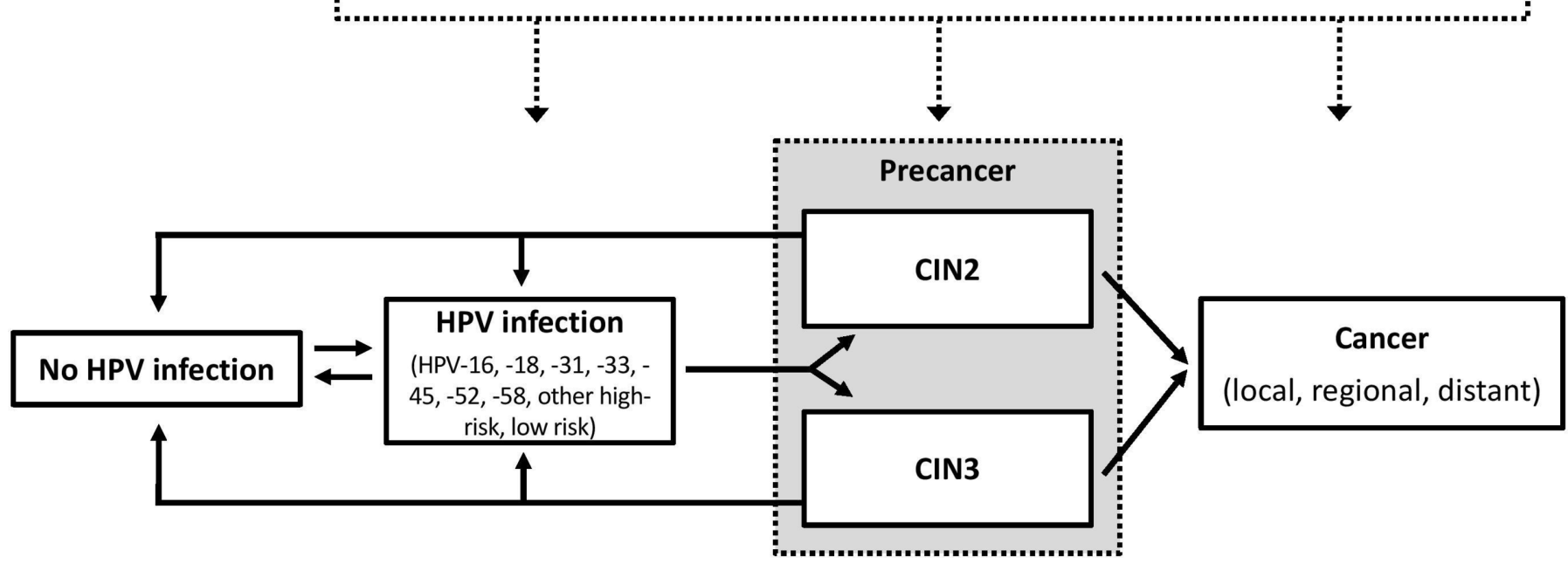

Figure 1 Model schematic. Screening, diagnosis and treatment of precancer or cancer are determined by screening strategy (this figure has been reproduced by permission of Oxford University Press, https://global.oup.com/academic/rights/ permissions/autperm/?cc=us\&lang=en\&). CIN2, cervical intraepithelial neoplasia grade 2; CIN3, cervical intraepithelial neoplasia grade 3.

mortality). Disease progression and regression, clinical events, and economic outcomes are tracked over the lifetime of each woman and aggregated over the cohort of one million women.

We used a likelihood-based approach (described in detail elsewhere) to calibrate the natural history model to epidemiological data from Uganda. ${ }^{10} 12$ Data on HPV incidence, ${ }^{17}$ progression and clearance, ${ }^{18}$ precancer progression $^{19}$ and regression, ${ }^{20}$ and cancer progression and mortality ${ }^{21-24}$ from longitudinal studies were used to establish baseline input values. Age-specific and type-specific HPV incidence, naturally acquired immunity following HPV infection, and progression and regression of precancer were identified as parameters with a high degree of uncertainty, and were thus selected for calibration. We set a range of plausible bounds for these uncertain model parameters and then randomly sampled from a uniform distribution across this range of values. ${ }^{10}{ }^{12}$ The range of plausible bounds that was sampled from for each variable is shown in the technical online supplementary appendix. Each model simulation selected one random value within the bounds for each uncertain parameter, creating a unique natural history input parameter set. By summing the log-likelihood of model-projected outcomes for each input parameter set relative to the epidemiological data on age-specific high-risk HPV prevalence and age-specific cancer incidence from Uganda, ${ }^{10}$ we computed a goodness-of-fit score. We selected the 50 top-fitting input parameter sets to use in this analysis. Results are reported as the mean and range across the top 50 parameter sets, and ICERs are reported as the ratio of the mean costs divided by the mean effects of one strategy versus another across sets. ${ }^{25}$

\section{Screening strategies}

We considered the following screening strategies (figure 2): (1) self-collected HPV testing at home or work followed by VIA triage for HPV-positive women, with cryotherapy for eligible women who were positive on both tests (HPV-VIA); (2) self-collected HPV testing at home or work followed by cryotherapy for all eligible HPV-positive women (HPV screen-and-treat strategy, HPV-ST) and (3) VIA at the clinic followed by cryotherapy for eligible women (VIA). While HPV-ST was not evaluated in the ASPIRE study, we considered this strategy over concerns that VIA triage of HPV-positive women may miss some precancer. The HPV-ST strategy is identical to the HPV-VIA strategy, except we assumed that all eligible HPV-positive women were referred to cryotherapy at Kisenyi clinic (regardless of visual inspection findings). In all three strategies, women who were not deemed eligible for cryotherapy at the Kisenyi clinic (based on visual inspection in all strategies) were referred to a tertiary care centre for further evaluation with colposcopy.

We evaluated screening once in a lifetime at age 39 to represent the average age and number of screens in the ASPIRE trial. In keeping with WHO guidelines to prioritise screening among women aged $30-49$ years, ${ }^{5}$ we also evaluated scenarios of screening three times at ages 30 , 40 and 50 years and screening every 5 years at ages 30,35 , 40,45 and 50 years. For the base case analysis, we assumed screening coverage of $70 \%$. Table 1 displays model inputs on screening uptake, compliance, test performance, treatment eligibility and treatment effectiveness. Values for screening uptake, the proportion of women who were reachable by phone for delivery of results, and compliance with Kisenyi Health Centre visits and cryotherapy 


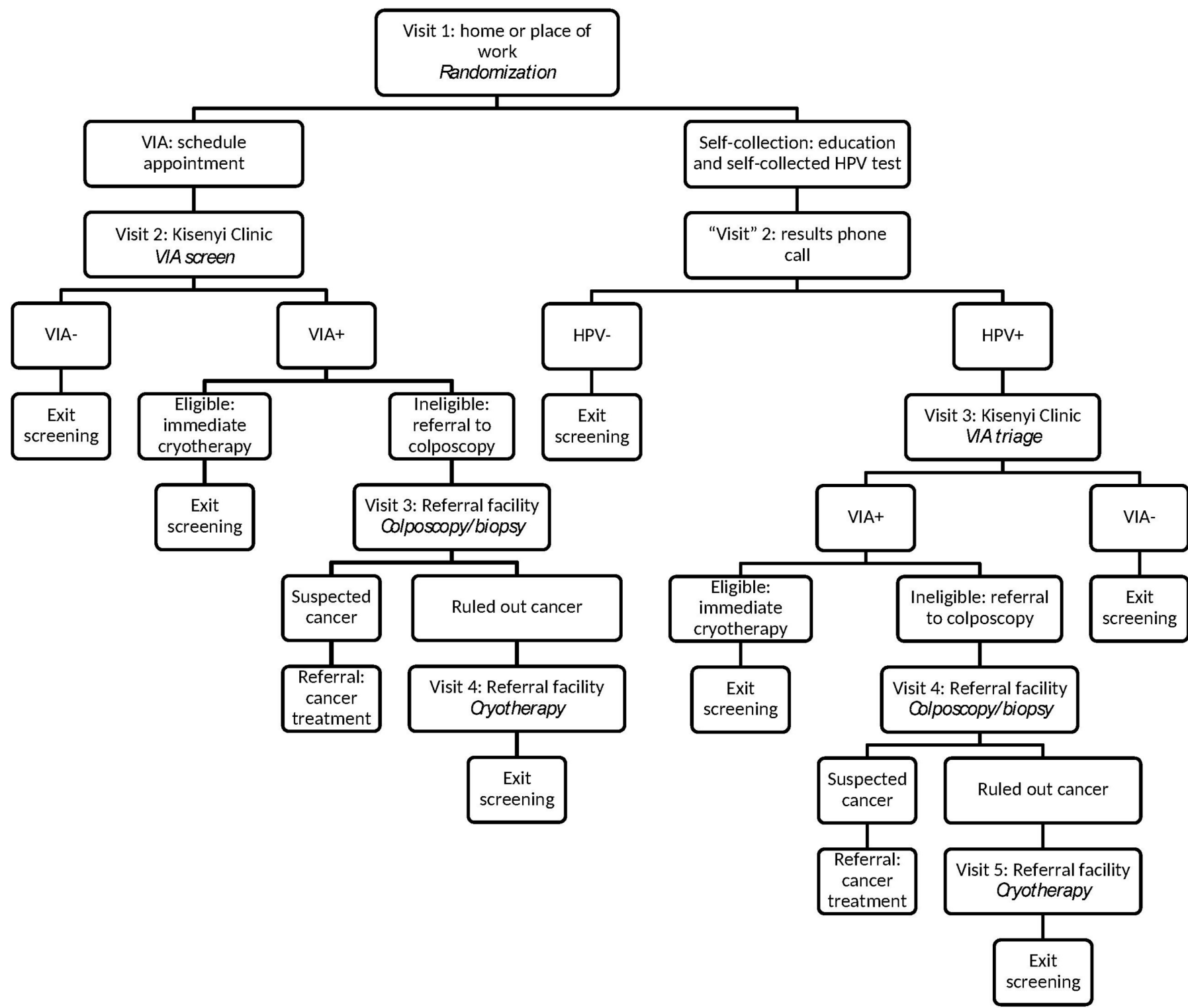

Figure 2 Pathways of care. This figure depicts the HPV-VIA and VIA screening strategies. The HPV-ST strategy is identical to HPV-VIA, except all HPV-positive women are offered cryotherapy (if eligible) or are referred to a tertiary care centre (if ineligible). ST, screen-and-treat strategy; VIA, visual inspection with acetic acid.

were informed by the ASPIRE trial. Compliance and uptake for the HPV-ST strategy was assumed to be identical to the HPV-VIA arm as the structure of the screening pathway was assumed to be the same. HPV test performance characteristics with self-collection were informed by a care HPV demonstration project in Uganda. ${ }^{6}$

\section{Cost data}

Cost data and time estimates are described extensively in the technical online supplementary appendix and presented in table 1. Costing was done from a societal perspective so that policy-makers can evaluate the impact on all stakeholders. ${ }^{26}$ Costs included direct medical costs (including CHW salary, provider wages, supply costs, sample transport costs, laboratory costs and equipment costs), women's time costs and programmatic costs. Briefly, cost data and time estimates were derived from a review of ASPIRE budget sheets, receipts, grant reconciliation sheets and consultation with researchers involved in the trial. When applicable, costs attributable to NGCT sampling were excluded. In several cases, ASPIRE cost data were not available and we instead used costs from other studies. HPV self-collection test cost, supplies, HPV laboratory costs and cryotherapy equipment costs were informed by the PATH START-UP demonstration project in Uganda. ${ }^{69}$ Cancer treatment costs were derived from Campos et al..$^{27}$ Costs were collected in US\$, Canadian dollars and Ugandan shillings (UGX). All costs are presented in 2014 US $\$$. If costs were collected in 2014, exchange rates were applied to convert costs to $2014 \mathrm{US} \$ .^{28}$ If costs were collected in a different year, we converted the currency to UGX, applied Ugandan GDP deflators to account for inflation, ${ }^{29}$ and then converted the cost to 
Table 1 Baseline values and ranges for sensitivity analysis ${ }^{*}$

\section{Baseline value}

Sensitivity analysis

Screening and treatment parameters

Screening age(s) $)^{511}$

1x: age 39 years;

$3 x$ : ages 30,40 and 50 years;

$5 x$ : ages $30,35,40,45$ and 50 years

Population coverage

Uptake of self-collected HPV testing ${ }^{11}$

Uptake of VIA ${ }^{11}$

Proportion of women successfully contacted over phone with HPV test results ${ }^{11}$

$70.0 \%$

$99.2 \%$

$48.4 \%$

Proportion of contacted HPV-positive women attending the $\quad 97.1 \%$ clinic $^{11}$

Compliance for all other visits

$85.0 \%$

Proportion of eligible women receiving cryotherapy following $78.6 \%$ positive VIA ${ }^{11}$

Proportion of eligible women receiving cryotherapy following $\quad 78.6 \%$ positive HPV test ${ }^{11}$
Proportion of women receiving treatment following
$85.0 \%$ colposcopy

Proportion of women who refuse cryotherapy then return at a $0.0 \%$ later date ${ }^{1137}$

Test sensitivity/specificity for CIN2+

HPV (self-collected) ${ }^{6}$

VIA (primary screen) ${ }^{6} 31$

$77.0 \% / 82.0 \%$

VIA (triage test) ${ }^{6} 3233$

$73.6 \% / 66.6 \%$

$73.6 \% / 66.6 \%$

$52.0 \%$

Test sensitivity/specificity for $\mathrm{CIN} 1+$, $\operatorname{colposcopy}^{10} \ddagger$

Eligibility for cryotherapy ${ }^{34-36}$

$\begin{array}{lll}\text { No lesion } & 90.0 \% & 72.2 \%-92.7 \% \\ \text { CIN2 } & 85.0 \% & 42.2 \%-87.7 \% \\ \text { CIN3 } & 75.0 \% & 42.2 \%-82.7 \% \\ \text { Cancer } & 10.0 \% & - \\ \text { Cryotherapy effectiveness at the clinic }^{38} 44 & 81.0 \% & 70.0 \%-92.0 \% \\ \text { Cryotherapy effectiveness at the referral centre }^{44} & 81.0 \% & 70.0 \%-92.0 \% \\ \text { Proportion of women who retain HPV infection following } & 10.0 \% & - \\ \text { cryotherapy } & & \end{array}$

Cost parameters, women's time costs

$\begin{array}{lll}\text { Women's time cost, per hour } & \text { US } \$ 0.46 & - \\ \text { Self-collection of HPV sample } & \text { US } \$ 0.19 & - \\ \text { VIA screen } & \text { US } \$ 1.38 & - \\ \text { Cryotherapy with no VIA (Kisenyi) } & \text { US\$1.46 } & - \\ \text { Extra time for cryotherapy post-VIA } & \text { US\$0.03 } & - \\ \text { Colposcopy and biopsy } & \text { US } \$ 2.76 & - \\ \text { Cryotherapy (Mulago National Referral Hospital) } & \text { US\$2.76 }\end{array}$

Cost parameters, programmatic costs $†$

Programmatic cost per women screened

US\$6.58

Cost parameters, direct medical costs $\S$

Self-collected HPV test ${ }^{101145}$

US\$12.73 
Table 1 Continued

\begin{tabular}{|c|c|c|}
\hline & Baseline value & Sensitivity analysis \\
\hline VIA & US\$14.64 & - \\
\hline Cost per cryotherapy ${ }^{1045}$ & US\$5.85 & US\$27.37 \\
\hline \multicolumn{3}{|l|}{ Treatment of cancer 273746} \\
\hline Local & US\$627 & US\$2328 \\
\hline Distant & US\$797 & US\$3953 \\
\hline
\end{tabular}

1x: screening at age 39; 3x: screening at ages 30, 40 and 50; 5x: screening at ages 30, 35, 40, 45 and 50.

${ }^{*}$ Costs are described in greater detail in the technical online supplementary appendix.

†Programmatic costs include costs for a programme assistant and a community preparedness campaign.

ҒAs CIN1 is not actually a health state in the model, colposcopy performance is based on the health state of no lesion, HPV infection, CIN2 or CIN3. A treatment threshold for CIN1 was estimated by weighting colposcopy sensitivity for HPV+ women based on the prevalence of CIN1 among HPV+ women in the Uganda START-UP study.

§Base case direct medical costs of HPV testing and cryotherapy were informed by START-UP ${ }^{10} 45$ due to the ASPIRE trial using HPV genotyping technology at an additional expense (this would not be performed in a non-research setting) and the number of assumptions required for cryotherapy cost amortisation.

ASPIRE, Advances in Screening and Prevention of Reproductive Cancers; CIN, cervical intraepithelial neoplasia; HPV, human papillomavirus; VIA, visual inspection with acetic acid.

US\$. Equipment costs were annualised to represent the duration of their use assuming a $3 \%$ interest rate.

Women's time spent travelling, waiting for and receiving care was valued using an average hourly wage for women enrolled in the trial. This was calculated by applying ASPIRE demographic survey data on education levels to Ugandan Ministry of Gender, Labour and Social Development data on average wages based on education level. ${ }^{30}$ Time estimates were informed by the ASPIRE demographic survey and consultation with researchers and clinicians who took part in the ASPIRE trial. Other societal costs, such as lost future productivity and impact on education have not been included due to a lack of data to objectively estimate these costs.

Programmatic costs were derived from the ASPIRE trial. These costs included a programme assistant and a community preparedness campaign that occurred in the month leading up to the trial to educate women on HPV and cervical cancer.

Further details on cost data and assumptions are provided in the technical online supplementary appendix.

\section{Sensitivity and scenario analyses}

To explore uncertainty around model inputs, we performed univariate and multivariate sensitivity analyses under the scenario of once in a lifetime screening at age 39 on the following variables: VIA primary screening test sensitivity/specificity, ${ }^{31}$ VIA triage test sensitivity, ${ }^{32}{ }^{33}$ cryotherapy eligibility, ${ }^{11}{ }^{34-36} \mathrm{HPV}$ test direct medical costs, ${ }^{11}$ cryotherapy treatment costs, ${ }^{11}$ cancer treatment costs, ${ }^{37}$ cryotherapy efficacy, ${ }^{38}$ the proportion of women who were reachable by phone for results delivery, ${ }^{11}$ midwife costs per VIA, CHW costs, loss to follow-up for referral centre visits, screening coverage, proportion of women who return for cryotherapy after initially refusing, colposcopy costs and discount rates.
Additionally, we performed the following scenario analyses: we evaluated only HPV-VIA and VIA once, three times, and five times per lifetime, ignoring the HPV-ST strategy that was inferred from ASPIRE results; we assumed all precancer treatment at referral centres was loop electrosurgical excisional procedure (LEEP) by increasing the treatment efficacy to $96.4 \%^{39}$ and the direct medical costs to US $\$ 86.70$ per LEEP (in the base case, only cryotherapy was available $)^{9}$; and we leveraged data from a study demonstrating that door-to-door follow-up with CHWs in South Africa increased adherence to cervical cancer screening visits and increased the CHW cost per women by US $\$ 2.68$ to increase compliance in all three strategies. ${ }^{40}$

\section{RESULTS}

\section{Reduction in cervical cancer risk}

The relative and absolute reductions in cancer risk for HPV-ST, HPV-VIA and VIA once per lifetime, three times per lifetime and five times per lifetime compared with no screening are presented in table 2 . At every screening frequency, HPV-ST led to the largest reduction in lifetime risk of cervical cancer, followed by HPV-VIA, and then VIA. Cancer risk reduction increased with the number of lifetime screens for all strategies. Screening every 5 years between ages 30 and 50 years reduced cancer risk by $42.8 \%, 26.1 \%$ and $24.2 \%$ for HPV-ST, HPV-VIA and VIA, respectively.

\section{Cost-effectiveness}

Results from cost-effectiveness analysis are presented in table 2 and figure 3. HPV-ST was the least costly and most effective strategy at all screening frequencies, dominating HPV-VIA and VIA. The ICERs of HPV-ST once, three times and five times per lifetime were US $\$ 130$ per YLS, US\$240 per YLS and US\$470 per YLS, respectively. 
Table 2 Base case results*

\begin{tabular}{llcl}
\hline & ICER (US\$/YLS) & $\begin{array}{l}\text { Relative cervical cancer risk } \\
\text { reductiont }\end{array}$ & $\begin{array}{l}\text { Absolute lifetime cervical } \\
\text { cancer risk }\end{array}$ \\
\hline No screening & - & - & $4.2 \%(3.8 \%-4.7 \%)$ \\
HPV-ST 1x & $130(110-150)$ & $15.0 \%(13.3 \%-16.6 \%)$ & $3.5 \%(3.2 \%-4.0 \%)$ \\
VIA 1x & DOM & $7.2 \%(6.3 \%-8.2 \%)$ & $3.9 \%(3.5 \%-4.3 \%)$ \\
HPV-VIA 1x & DOM & $7.6 \%(6.7 \%-8.7 \%)$ & $3.9 \%(3.5 \%-4.3 \%)$ \\
HPV-ST 3x & 240 (210-280) & $33.0 \%(30.6 \%-35.5 \%)$ & $2.8 \%(2.4 \%-3.1 \%)$ \\
VIA 3x & DOM & $16.9 \%(15.4 \%-18.8 \%)$ & $3.5 \%(3.1 \%-3.9 \%)$ \\
HPV-VIA 3x & DOM & $18.4 \%(16.7 \%-20.5 \%)$ & $3.4 \%(3.0 \%-3.8 \%)$ \\
HPV-ST 5x & $470(410-550)$ & $42.8 \%(39.8 \%-45.6 \%)$ & $2.4 \%(2.1 \%-2.7 \%)$ \\
VIA 5x & DOM & $24.2 \%(22.0 \%-26.4 \%)$ & $3.2 \%(2.8 \%-3.5 \%)$ \\
HPV-VIA 5x & DOM & $26.1 \%(23.9 \%-28.5 \%)$ & $3.1 \%(2.7 \%-3.4 \%)$ \\
\hline
\end{tabular}

The base case analysis compares all three screening strategies at all three screening frequencies. $1 \mathrm{x}$ : screening at age 39 years;

$3 x=$ screening at ages 30,40 and 50 years; $5 x=$ screening at ages $30,35,40$, 45 and 50 years; DOM: more costly and less effective or having a higher ICER than equally or more effective strategies; ICER: expressed in 2014 US\$ per YLS.

*Values indicate the mean results across the top 50 best fitting parameter sets. The minimum and maximum values across these 50 parameter sets are shown in parentheses.

†Relative reduction in lifetime risk of cervical cancer is compared with no screening.

DOM, dominated strategy; HPV, human papillomavirus; HPV-ST, community-based HPV self-collection with clinic-based cryotherapy of eligible HPV+ women; HPV-VIA, community-based HPV self-collection with clinic-based VIA triage and immediate treatment of eligible VIA+ women; ICER, incremental cost- effectiveness ratio; ST, screen-and-treat strategy; VIA, visual inspection with acetic acid; VIA, clinic-based VIA with immediate treatment of eligible VIA+ women; YLS, years of life saved.

HPV-ST would therefore be very cost-effective based on a willingness to pay threshold of Uganda's 2014 GDP per capita (US\$730). ${ }^{14}$

\section{Sensitivity and scenario analysis}

Results from sensitivity and scenario analyses are described in tables 3 and 4 . The finding that the HPV-ST strategy was the most effective was robust as we varied VIA primary screening test performance, VIA triage test performance, cryotherapy eligibility, cryotherapy efficacy, telephone compliance, loss to follow-up for tertiary care centre visits, screening coverage, proportion of women who return for cryotherapy after initially refusing, direct medical costs of HPV testing and VIA,

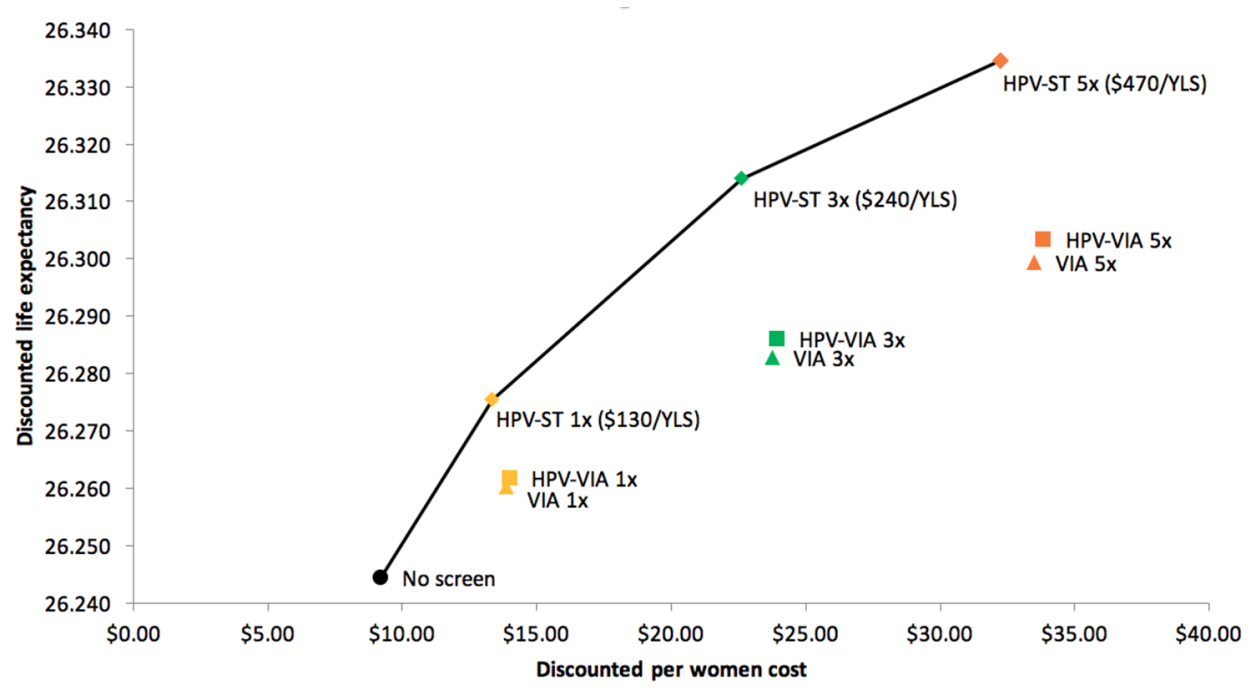

Figure 3 Cost-effectiveness results: base case analysis. ICERs (expressed in 2014 US\$ per year of life saved) are in parentheses for non-dominated strategies. 1x: screening at age 39 tears (yellow); 3x: screening at ages 30,40 and 50 years (green); 5x: screening at ages 30, 35, 40, 45 and 50 years (orange); HPV-ST: HPV screen and treat (diamonds); HPV-VIA: HPV with VIA triage (squares); VIA: VIA screen and treat (triangles). The black line denotes the efficiency frontier. Any strategy lying to the right of the efficiency frontier is a dominated strategy because it is more costly and less effective or has a higher ICER than equally or more effective strategies. ICER, incremental cost-effectiveness ratio; ST, screen-and-treat strategy; VIA, visual inspection with acetic acid; YLS, year of life saved. 
Table 3 Results: sensitivity analyses, once in a lifetime screening ${ }^{*}$

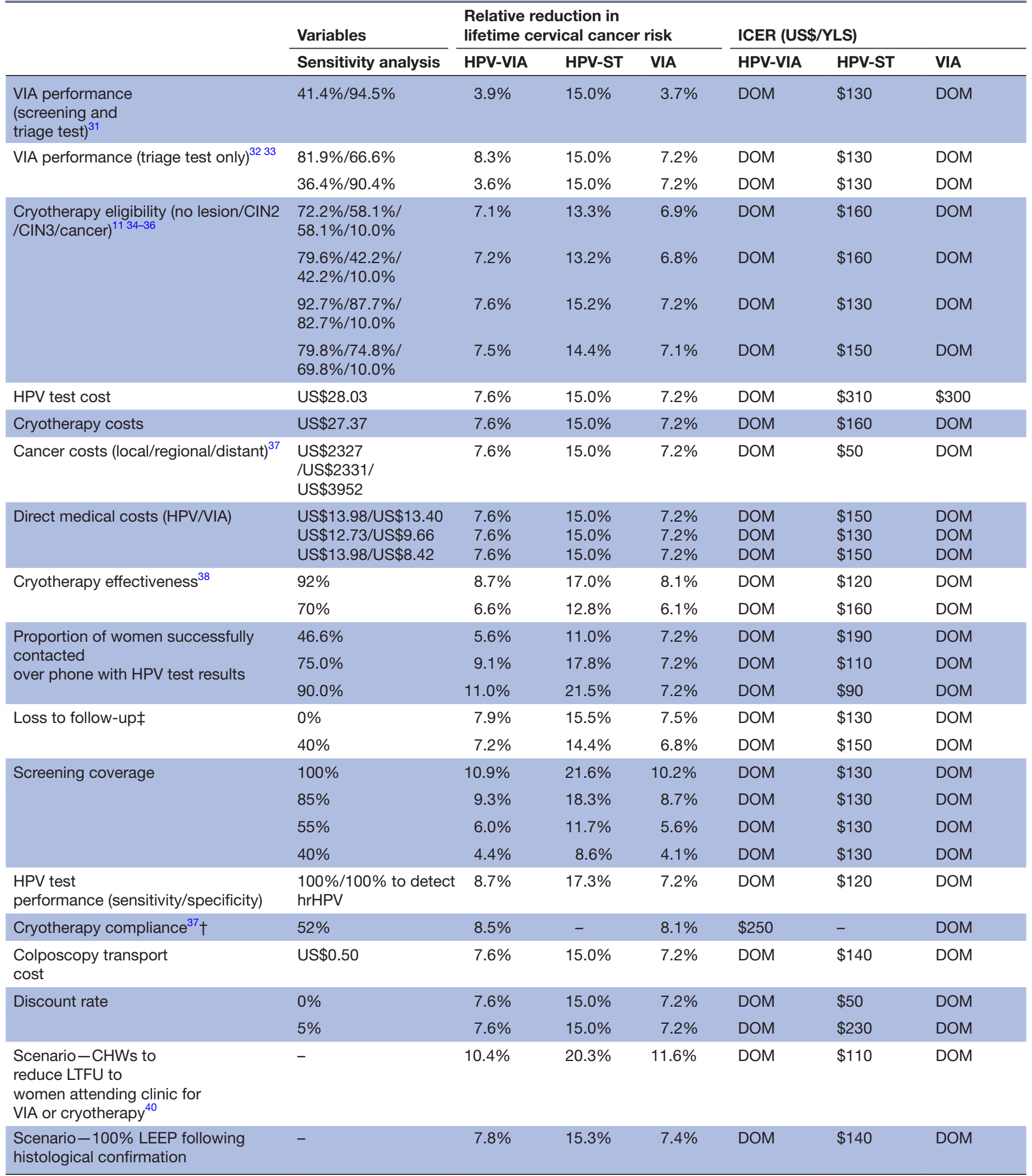

Relative reduction in lifetime cancer risk is relative to no screening; incremental cost-effectiveness ratios are presented in 2014 US\$; DOM: more costly or higher ICER than equally or more effective strategies.

*Sensitivity analyses are described in detail in the technical online supplementary appendix.

†The model was not equipped to handle a change in this variable in the HPV-ST arm.

$\ddagger$ This variable refers only to loss to follow up for visits to the referral centre for colposcopy or follow-up of cryotherapy ineligible lesions.

$\mathrm{CHW}$, community health worker; CIN2, cervical intraepithelial neoplasia grade 2; DOM, dominated strategy; ICER, incremental cost-

effectiveness ratio; LEEP, loop electrosurgical excisional procedure; LTFU, loss to follow-up; ST, screen-and-treat strategy; VIA, visual

inspection with acetic acid; YLS; year of life saved. 


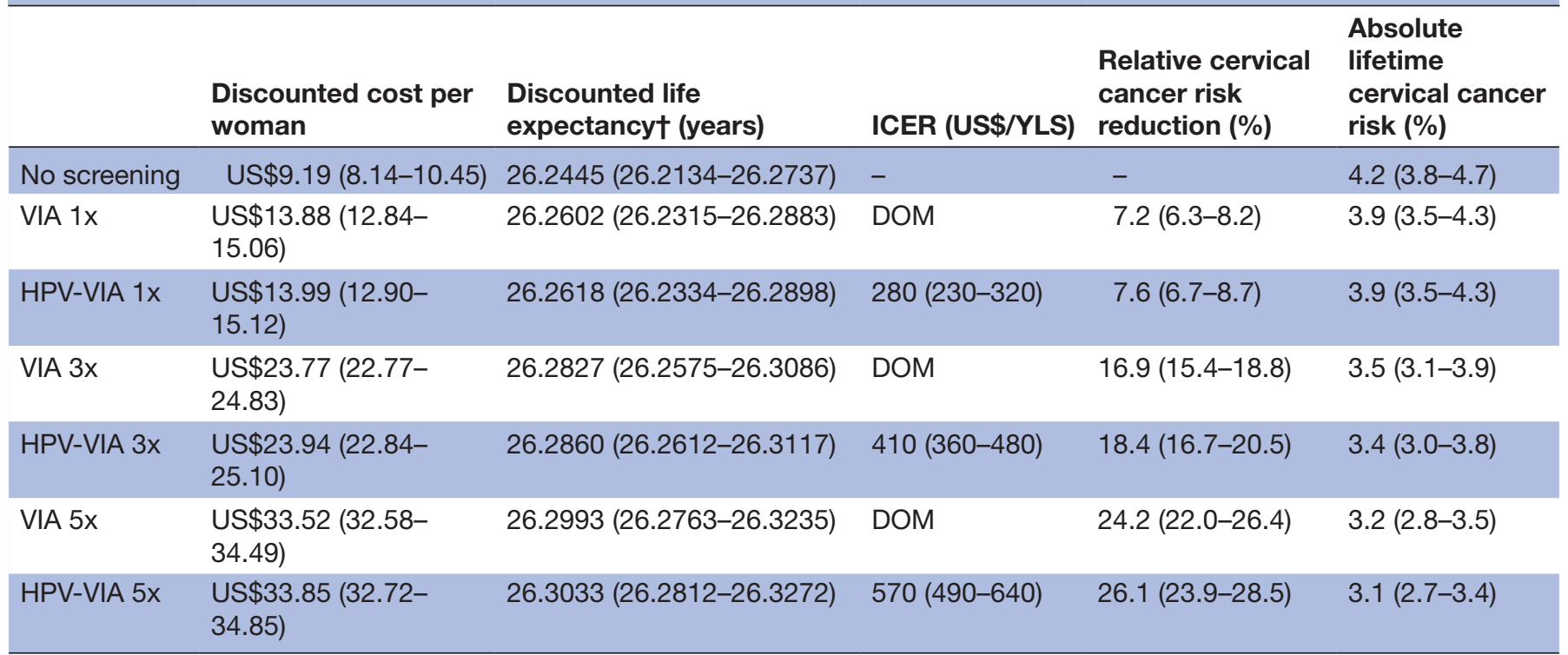

1x: screening at age 39 years; 3x: screening at ages 30,40 and 50 years; $5 x$ : screening at ages 30, 35, 40, 45 and 50 years; HPV-VIA: HPV with VIA triage; VIA: VIA screen and treat. DOM: more costly or higher ICER than equally or more effective strategies; incremental costeffectiveness ratio (ICER): expressed in 2014 US\$ per year of life saved (YLS); relative cancer reduction is the lifetime reduction relative to no screening.

*Values indicate the mean results across the top 50 top-fitting parameter sets. The minimum and maximum values across these 50 parameter sets are shown in parentheses.

†Discounted life expectancy is after age 9, the age at which women enter the model.

DOM, dominated strategy; ICER, incremental cost- effectiveness ratio; VIA, visual inspection with acetic acid; YLS, year of life saved.

and discount rates. HPV-VIA was more effective than VIA in all sensitivity analyses conducted except when telephone contact rates of HPV-positive women were reduced to $46.6 \%$ in the HPV-VIA strategy or the sensitivity of the VIA triage test was $36.4 \%$. The effectiveness of different strategies was most sensitive to changes in screening coverage and compliance variables. For example, increasing screening coverage to $100 \%$ led to an increase in the relative reduction of lifetime cervical cancer risk from $15.0 \%$ to $21.6 \%$ (HPV-ST), while increasing telephone contact rates to $90 \%$ increased relative reduction of lifetime cervical cancer risk from $15.0 \%$ to $21.5 \%$ (HPV-ST).

The HPV-ST strategy was very cost-effective and dominated HPV-VIA and VIA in all sensitivity analyses, except when HPV test costs were more than quadrupled, in which case VIA was no longer a dominated strategy and had an ICER of US\$300 per YLS. The ICER for HPV-ST fell below US $\$ 50$ per YLS when cancer costs increased more than threefold and when there was no discounting of future costs and life years, and only increased above US\$200 per YLS when HPV test costs increased or the discount rate was $5 \%$ (base case: US $\$ 130$ per YLS). Scenario analyses demonstrated that using CHWs for in-person follow-up could be a very cost-effective approach to increase retention to screening pathways, while using LEEP instead of cryotherapy had little impact on both cancer reductions and ICERs compared with the base case.
When we assumed only HPV-VIA and VIA were available (table 4), as in the ASPIRE study, HPV-VIA dominated VIA at all screening frequencies and would be very cost-effective with an ICER of US $\$ 570 /$ YLS when performed five times per lifetime.

\section{Patient and public involvement}

There was no patient or public involvement in this study as it was based on mathematical modelling. However, the ASPIRE trial held multiple community engagement and education workshops to describe the interventions and provide broader education about HPV and cervical cancer in Uganda. The CHWs who were hired in the trial were members of the local community and were trained on the trial protocol and procedures. While all individual screening results were communicated with trial participants, there was no formal process for relaying the trial results to the participants. Trial results were disseminated to the local study team.

\section{DISCUSSION}

We evaluated the cost-effectiveness of a novel self-collected HPV testing strategy using uptake, follow-up and cost data from the ASPIRE trial in Uganda. To our knowledge, this is the first study to use trial data to evaluate the cost-effectiveness of a delivery model relying on CHWs to offer home-based HPV self-collection. We found that screening with self-collected HPV testing followed by cryotherapy for eligible HPV-positive women (HPV-ST) 
would be very cost-effective in Uganda and could reduce the risk of cervical cancer by $15.0 \%$ if offered once in a lifetime at age 39 or up to $42.8 \%$ if offered every 5 years between ages 30 and 50 years. HPV testing with VIA triage of HPV-positive women to determine treatment and VIA alone were both more costly and less effective than HPV-ST, and thus not efficient strategies.

Previous cost-effectiveness studies evaluating self-collected HPV testing have demonstrated that self-collection can be a cost-effective screening method if it achieves higher levels of population coverage than provider-collected HPV testing. ${ }^{4}$ For instance, in weighing the trade-off between test sensitivity and higher screening coverage, Campos et al found that a $20 \%$ loss in test sensitivity due to self-collection can be offset by a $20 \%$ gain in screening coverage. ${ }^{8}$ While the ASPIRE trial compared HPV self-collection to VIA, the results still demonstrate the importance of uptake rates in determining cost-effectiveness. In the ASPIRE trial, 248 out of 250 women agreed to self-collect, while 121 out of 250 women attended the clinic for VIA. This enhanced uptake of HPV self-collection, when combined with the higher sensitivity to detect precancer of self-collected HPV testing (compared with VIA), rendered the HPV-ST screening strategy the most effective and cost-effective screening strategy in this analysis. These findings provide evidence that real-world increases in screening coverage due to door-to-door self-collection opportunities may translate into improved health and cost-effectiveness outcomes, and were robust to numerous sensitivity analyses.

The ASPIRE self-collection strategy could be further improved on if more HPV-positive women were successfully contacted with their results. In the ASPIRE trial, with a protocol of three phone attempts to contact women with their results, 34 of the 54 women who were HPV-positive could not be contacted. Of the HPV-positive women who were successfully contacted, 33 out of the 34 came in to Kisenyi clinic for follow-up. Strategies to improve delivery of test results should be explored. Sensitivity analysis showed that increasing delivery of results would further decrease cancer risk, and that having CHWs do in-person follow-up could be a cost-effective strategy to improve management for screen-positive women. ${ }^{40}$ Alternatively, other models of community-based self-collection have been proposed. Campos et al showed how a community mobilisation campaign with group self-collection is a cost-effective alternative to provider collection if it increases population coverage. ${ }^{9}$ Future research should compare different models of offering self-collection and consider setting-specific factors that could make certain delivery methods more efficient.

An important finding is that HPV testing is more effective when there is no VIA triage before cryotherapy, a result that contradicts current WHO recommendations for countries with enough resources to provide a sequence of tests. ${ }^{5}$ This is due to the high false negative rate of VIA, the implications of which are amplified when the false negatives are in a high-risk group (ie, women already known to be HPV-positive). Our base case analysis assumed that VIA as a triage test performed similarly to VIA in a general screening population, detecting $73.6 \%$ of CIN2+. ${ }^{6}$ Other studies in Africa looking at VIA sensitivity as a triage test have showed large variability, with sensitivities ranging from $25.0 \%$ to $81.9 \% .^{32} 41$ These findings suggest that future programmes should consider incorporating HPV-ST. However, this will lead to an increase in the number of cryotherapy procedures in women who may not have or may not develop precancer. While our results demonstrated that ST would be cost-effective, LMICs will likely face human resource and capacity constraints and may be overburdened by the high number of cryotherapy procedures. Moreover, while cryotherapy has been shown to be safe and acceptable, ${ }^{38}$ concern has arisen that there could be an increased risk of HIV incidence following cryotherapy. ${ }^{42}$ This is of particular concern in settings with high HIV prevalence. Better triage tests are needed to improve identification of women at high risk of developing cervical cancer while reducing the number of treatments that are required of overburdened health systems.

Several limitations of this study should be noted. Our costing of the ASPIRE trial was hindered by the fact that the trial was not initially designed to evaluate cost-effectiveness, and thus all cost estimates are retrospective. Therefore, the trial may not reflect real-world (as opposed to study) conditions, and thus the amount paid for equipment, supplies and labour does not necessarily reflect actual programmatic costs. Cancer treatment costs had to be inferred from other studies. In a few cases, we used cost data from the PATH START-UP demonstration project to more accurately reflect real-world costs, as detailed in the technical online supplementary appendix. The base case assumption that VIA was more costly than HPV testing is unusual, and attributable to VIA having a provider cost (where HPV testing was self-collected) and requiring more $\mathrm{CHW}$ time to encourage compliance. Still, sensitivity analyses revealed that HPV-ST remained the dominant screening strategy even as the relative cost of HPV testing versus VIA was varied considerably. Concern has been raised that using GDP per capita as a threshold for cost-effectiveness may not be affordable in LMICs, however, even with a threshold of $50 \%$ of GDP per capita our analysis shows that HPV-ST one and three times per lifetime would be very cost-effective. ${ }^{43}$ Furthermore, the costing for this study was based on a trial involving 250 women in each arm, which may not be sufficient to capture programmatic economies of scale.

In addition to limitations on costing data, the trial setting limited the scope of this cost-effectiveness analysis. It is unclear how real-world human resource constraints might affect the cost-effectiveness of the different screening strategies through impact on women's waiting time in typical primary care settings. Furthermore, decision-makers will need to assess where limited healthcare provider time is best spent. It is of note that all the women in this study had access to a mobile phone. This may not be the case in rural and remote areas of Uganda, so novel 
screening approaches may need to be developed that would be more suitable for these locations.

While it was not a stated objective of this study to evaluate the cost-effectiveness of NGCT screening, this was an integral part of the ASPIRE trial due to the hypothesis that efficiency gains could be achieved by bundling health interventions with overlapping infrastructure needs. Moving forward, developing models that can evaluate the integrated delivery of primary care services will be critical to assess the wider impacts of new healthcare delivery methods in low-resource settings, as well as to capture potential synergies associated with packaging interventions.

In 2012, over 230000 women in LMICs died from cervical cancer, approximately 2200 of whom were from Uganda. ${ }^{2}$ This study demonstrates that there are very cost-effective options that could significantly reduce morbidity and mortality attributable to cervical cancer. Implementation studies on a larger scale-that would assess the effectiveness and cost-effectiveness of community-based screening in the context of the Ugandan health system-are warranted. Such studies will provide lessons for nascent screening programmes in low-resource settings with a high burden of cervical cancer.

\section{Author affiliations}

${ }^{1}$ Faculty of Medicine, University of British Columbia, Vancouver, British Columbia, Canada

${ }^{2}$ Women's Health Research Institute, British Columbia Women's Hospital and Health Centre, Vancouver, British Columbia, Canada

${ }^{3}$ Department of Health Policy and Management, Center for Health Decision Science, Harvard T.H. Chan School of Public Health, Boston, Massachusetts, USA

${ }^{4}$ Department of Obstetrics and Gynecology, University of British Columbia, Vancouver, British Columbia, Canada

${ }^{5}$ Department of Obstetrics and Gynecology, Makerere University, Kampala, Uganda ${ }^{6}$ Department of Obstetrics and Gynecology, Mulago National Referral Hospital, Kampala, Uganda

${ }^{7}$ School of Population and Public Health, University of British Columbia, Vancouver, British Columbia, Canada

${ }^{8}$ Centre for Health Evaluation and Outcome Sciences, St. Paul's Hospital, Vancouver, British Columbia, Canada

Contributors All authors were involved in the design of the research study, critically assessed the content and agreed with the final decision to publish. AKM and NGC drafted the manuscript. AKM, NGC, CR, SS and JJK conducted the statistical analysis. HNP, AKM and NGC drafted the figures. HNP, JB, MS, SMM-F, JS, HA, AR and GSO designed and ran the ASPIRE trial.

Funding This work was supported by the Canadian Institute for Health Research (ClHR), grant number FDN-143339.

Competing interests None declared.

Patient consent Not required.

Ethics approval University of British Columbia and Makerere University.

Provenance and peer review Not commissioned; externally peer reviewed.

Data sharing statement All relevant data are presented in the manuscript and technical appendix.

Open access This is an open access article distributed in accordance with the Creative Commons Attribution Non Commercial (CC BY-NC 4.0) license, which permits others to distribute, remix, adapt, build upon this work non-commercially, and license their derivative works on different terms, provided the original work is properly cited and the use is non-commercial. See: http://creativecommons.org/ licenses/by-nc/4.0/ (c) Article author(s) (or their employer(s) unless otherwise stated in the text of the article) 2018. All rights reserved. No commercial use is permitted unless otherwise expressly granted.

\section{REFERENCES}

1. Kitchener HC, Castle PE, Cox JT. Chapter 7: Achievements and limitations of cervical cytology screening. Vaccine 2006;24(Suppl 3):S63-S70.

2. Ferlay J, Soerjomataram I, Ervik M, et al. GLOBOCAN 2012 v1.0, Cancer Incidence and Mortality Worldwide: IARC CancerBase No.11 [internet]. Lyon, France: International Agency for Research on Cancer, 2013. http://globocan.iarc.fr

3. Bruni L, Diaz M, Barrionuevo-Rosas L, et al. Global estimates of human papillomavirus vaccination coverage by region and income level: a pooled analysis. Lancet Glob Health 2016;4:e453-e463.

4. Mezei AK, Armstrong HL, Pedersen HN, et al. Cost-effectiveness of cervical cancer screening methods in low- and middle-income countries: A systematic review. Int J Cancer 2017;141:437-46.

5. World Health Organization. WHO guidelines for screening and treatment of precancerous lesions for cervical cancer prevention. Geneva: World Health Organization, 2013.

6. Jeronimo J, Bansil P, Lim J, et al. A multicountry evaluation of careHPV testing, visual inspection with acetic acid, and papanicolaou testing for the detection of cervical cancer/nt $J$ Gynecol Cancer 2014;24:576-85.

7. Teng FF, Mitchell SM, Sekikubo M, et al. Understanding the role of embarrassment in gynaecological screening: a qualitative study from the ASPIRE cervical cancer screening project in Uganda. BMJ Open 2014:4:e004783.

8. Campos NG, Castle PE, Wright TC, et al. Cervical cancer screening in low-resource settings: A cost-effectiveness framework for valuing tradeoffs between test performance and program coverage. Int $J$ Cancer 2015;137:2208-19.

9. Campos NG, Tsu V, Jeronimo J, et al. Cost-effectiveness of an HPV self-collection campaign in Uganda: comparing models for delivery of cervical cancer screening in a low-income setting. Health Policy Plan 2017;32:956-68.

10. Campos NG, Tsu V, Jeronimo J, et al. When and how often to screen for cervical cancer in three low- and middle-income countries: A cost-effectiveness analysis. Papillomavirus Res 2015;1:38-58.

11. Moses E, Pedersen HN, Mitchell SM, et al. Uptake of communitybased, self-collected HPV testing vs. visual inspection with acetic acid for cervical cancer screening in Kampala, Uganda: preliminary results of a randomised controlled trial. Trop Med Int Health 2015;20:1355-67.

12. Campos NG, Burger EA, Sy S, et al. An updated natural history model of cervical cancer: derivation of model parameters. Am J Epidemiol 2014;180:545-55.

13. Kim JJ, Kuntz KM, Stout NK, et al. Multiparameter calibration of a natural history model of cervical cancer. Am J Epidemiol 2007;166:137-50.

14. United Nations Statistics Divison. Country profile: Uganda In: World Statistics Pocketbook. http://data.un.org/CountryProfile.aspx? crName=uganda (accessed 15 Apr 2017).

15. World Health Organization (WHO). Macroeconomics and Health: Investing in Health for Economic Development: Report on the Commission on Macroeconomics and Health. Geneva: World Health Organization, 2001. (accessed 10 Apr 2017).

16. World Health Organization. Making choices in health: WHO guide to cost-effectiveness analysis. Geneva: World Health Organization, 2003. http://www.who.int/choice/publications/p_2003_generalised_ cea.pdf (accessed 10 Apr 2017).

17. Muñoz N, Méndez F, Posso H, et al. Incidence, duration, and determinants of cervical human papillomavirus infection in a cohort of Colombian women with normal cytological results. J Infect Dis 2004;190:2077-87.

18. Herrero R, Hildesheim A, Rodríguez AC, et al. Rationale and design of a community-based double-blind randomized clinical trial of an HPV 16 and 18 vaccine in Guanacaste, Costa Rica. Vaccine 2008;26:4795-808.

19. McCredie MR, Sharples KJ, Paul C, et al. Natural history of cervical neoplasia and risk of invasive cancer in women with cervical intraepithelial neoplasia 3: a retrospective cohort study. Lancet Oncol 2008;9:425-34.

20. Keefe KA, Schell MJ, Brewer C, et al. A randomized, double blind, Phase III trial using oral beta-carotene supplementation for women with high-grade cervical intraepithelial neoplasia. Cancer Epidemiol Biomarkers Prev 2001;10:1029-35. 
21. Myers ER, McCrory DC, Nanda K, et al. Mathematical model for the natural history of human papillomavirus infection and cervical carcinogenesis. Am J Epidemiol 2000;151:1158-71.

22. McCrory D, Matchar D, Bastian L, et al. Evaluation of Cervical Cytology: Summary Rockville. Agency for Health Care Policy and Research Evidence Report Summaries, 1999 Feb.

23. Ries L, Eisner M, Kosary C, et al; SEER cancer statistics review, 1975-2001. Bethesda, MD: National Cancer Institute, 2004.

24. Surveillance Epidemiology and End Results (SEER) Program, National Cancer Institute, Division of Cancer Control and Population Sciences. SEER*Stat Database: Incidence-SEER 18 Regs Research Data + Hurricane Katrina Impacted Louisiana Cases Nov 2011 Sub (1973-2009 Varying) - Linked to County Attributes - Total U.S., 1969-2010 Counties. Bethesda, MD: National Cancer Institute, Division of Cancer Control and Population Sciences, Surveillance Research Program, Surveillance Systems Branch, 2012.

25. Stinnett $A A$, Paltiel $A D$. Estimating $C E$ ratios under second-order uncertainty: the mean ratio versus the ratio of means. Med Decis Making 1997;17:483-9.

26. Neumann PJ, Sanders GD, Russell LB, et al. Cost-effectiveness in health and medicine, second edition. Oxford University Press, 2016.

27. Campos NG, Kim JJ, Castle PE, et al. Health and economic impact of HPV 16/18 vaccination and cervical cancer screening in Eastern Africa. Int J Cancer 2012;130:2672-84.

28. The World Bank Group. Official exchange rate (LCU per US\$, period average). http://data.worldbank.org/indicator/PA.NUS.FCRF (accessed 10 Dec 2016).

29. The World Bank Group. GDP deflator. http://data.worldbank.org/ indicator/NY.GDP.DEFL.ZS (accessed December 10, 2016).

30. Ugandan Ministry of Gender Labour and Social Development. Labour market information status report for Uganda, 2006. http://www. afristat.org/contenu/pdf/Imis/lmis_status_ug.pdf.

31. Qiao YL, Sellors JW, Eder PS, et al. A new HPV-DNA test for cervicalcancer screening in developing regions: a cross-sectional study of clinical accuracy in rural China. Lancet Oncol 2008;9:929-36.

32. Muwonge R, Wesley RS, Nene BM, et al. Evaluation of cytology and visual triage of human papillomavirus-positive women in cervical cancer prevention in India. Int J Cancer 2014;134:2902-9.

33. Tebeu PM, Fokom-Domgue J, Crofts V, et al. Effectiveness of a twostage strategy with HPV testing followed by visual inspection with acetic acid for cervical cancer screening in a low-income setting. Int J Cancer 2015;136:E743-E750.

34. Gage JC, Rodriguez AC, Schiffman M, et al. An evaluation by midwives and gynecologists of treatability of cervical lesions by cryotherapy among human papillomavirus-positive women. Int $\mathrm{J}$ Gynecol Cancer 2009;19:728-33.

35. Gage JC, Rodriguez AC, Schiffman M, et al. Treatability by cryotherapy in a screen-and-treat strategy. J Low Genit Tract Dis 2009;13:174-81.

36. Mgomella G, Chikamata D, Lucas E, et al. Prevention of cervical cancer through screening using visual inspection with acetic acid (VIA) and treatment with cryotherapy, a demonstration project in six African countries: Malawi, Madagascar, Nigeria, Uganda,the United Republic of Tanzania, and Zambia. World Health Organization. 2012.

37. Nelson S, Kim J, Wilson FA, et al. Cost-Effectiveness of Screening and Treatment for Cervical Cancer in Tanzania: Implications for other Sub-Saharan African Countries. Value Health Reg Issues 2016;10:1-6

38. Sauvaget C, Muwonge R, Sankaranarayanan R. Meta-analysis of the effectiveness of cryotherapy in the treatment of cervical intraepithelial neoplasia. Int J Gynaecol Obstet 2013;120:218-23.

39. Chirenje ZM, Rusakaniko S, Akino V, et al. A randomised clinical trial of loop electrosurgical excision procedure (LEEP) versus cryotherapy in the treatment of cervical intraepithelial neoplasia. J Obstet Gynaecol 2001;21:617-21.

40. Goldhaber-Fiebert JD, Denny LA, De Souza M, et al. Program spending to increase adherence: South African cervical cancer screening. PLoS One 2009;4:e5691.

41. Bigoni J, Gundar M, Tebeu PM, et al. Cervical cancer screening in sub-Saharan Africa: a randomized trial of VIA versus cytology for triage of HPV-positive women. Int J Cancer 2015;137:127-34.

42. Denny L, Kuhn L, Hu CC, et al. Human papillomavirus-based cervical cancer prevention: long-term results of a randomized screening trial. J Natl Cancer Inst 2010;102:1557-67.

43. Robinson LA, Hammitt JK, Chang AY, et al. Understanding and improving the one and three times GDP per capita cost-effectiveness thresholds. Health Policy Plan 2017;32:141-5.

44. Olatunbosun OA, Okonofua FE, Ayangade SO. Outcome of cryosurgery for cervical intraepithelial neoplasia in a developing country. Int J Gynaecol Obstet 1992;38:305-10.

45. Mvundura M, Tsu V. Estimating the costs of cervical cancer screening in high-burden Sub-Saharan African countries. Int J Gynaecol Obstet 2014;126(2):151-5

46. Goldie SJ, Gaffikin L, Goldhaber-Fiebert JD, et al. Cost-effectiveness of cervical-cancer screening in five developing countries. $N$ Engl $J$ Med 2005;353:2158-68. 